This article was published in Archives of Virology, 159, 191-198, 2014 http://dx.doi.org/10.1007/s00705-013-1801-9

\title{
Bovine papillomavirus: opening new trends for comparative pathology
}

\section{Brief review}

\author{
Rui M. Gil da Costa $^{1,2^{*}}$, Rui Medeiros ${ }^{3,4,5,6}$
}

1- Laboratory for Process, Environmental and Energy Engineering (LEPAE), Chemical Engineering Dept., Faculty of Engineering, University of Porto (FEUP), Rua Dr. Roberto Frias, 4200-465 Porto, Portugal.

2- Experimental Pathology and Therapeutics Group, CI-IPOP, Portuguese Institute of Oncology, Rua Dr. António Bernardino de Almeida, 4200-072 Porto, Portugal.

3- Molecular Oncology GRP-CI and Virology LB, Portuguese Institute of Oncology, Rua Dr. António Bernardino de Almeida, 4200-072 Porto, Portugal.

4- ICBAS, Abel Salazar Institute for the Biomedical Sciences, University of Porto, Rua Jorge Viterbo Ferreira 228, 4050-313 Porto, Portugal.

5-CEBIMED, Faculty of Health Sciences, Fernando Pessoa University, Praça 9 de Abril 349, 4249-004 Porto, Portugal.

6- LPCC, Portuguese League Against Cancer (NRNorte), Research Dept., Estrada Interior da Circunvalação 6657, 4200-177 Porto, Portugal.

* Corresponding author:

Rui M. Gil da Costa

LEPAE, Chemical Engineering Dept., Faculty of Engineering, University of Porto, Rua Dr. Roberto Frias 4200-465 Porto, Portugal. rmcosta@fe.up.pt,

Phone: +351 225081440, Fax: +351225081440. 
Abstract- For many years, research on bovine papillomavirus (BPV) has contributed to the understanding of papillomavirus-induced pathology in humans and animals. The present review shows how recent studies on BPV keep providing evidence concerning key points in viral infection, such as the expression of viral proteins in lymphocytes and the occurrence of productive infections of the placenta. Studies on BPV-induced tumours also provide important information concerning the mechanisms of oncogenesis and immune evasion, as the cases of connexin 43 down-regulation with loss of intercellular gap junctions and Toll-like receptor 4 (TLR4) down-regulation in equine sarcoids. The biological functions of viral proteins are also being further clarified as in the case of E2, which was recently shown to load BPV genomes into host chromosomes during the S-phase, a process mediated by the ChlR1 protein. In the near future, the ongoing efforts to characterize and classify additional emerging BPV types are likely to broaden even further the possibilities for research.

\section{Introduction}

Animal papillomavirus such as the bovine papillomavirus (BPV), the cottontail rabbit papillomavirus and the canine oral papillomavirus have long been known to induce tumours in their host species. In fact, these viruses first provided evidence for virallyinduced carcinogenesis and have since been used as models to study the biology and pathology of human papillomavirus (HPV), as reviewed by Campo [1]. Papillomavirus are highly species-specific, making animal models for HPV difficult to obtain, except by using animal papillomavirus. Even though these difficulties in finding experimental models of HPV infection and carcinogenesis have been largely overcome during the first years of the new century, with the development of HPV-transfected organotypic cell cultures [2], research on animal papillomavirus, and particularly on BPV, does not seem to have diminished since then.

This review will deal with selected recent literature on animal papillomavirus, especially with those papers published over the last two years. Emphasis will be placed on BPV, since it causes several important syndromes and great losses among infected animals. Urinary bladder tumours associated with BPV infection caused losses worth over 4 million euros on state compensations and insurances paid to affected milk producers in the Azores between 2000 and 2006 [3], though this may be an underestimate. Research on BPV has led to most of the recent breakthroughs on the biology and pathology of animal papillomavirus, with major implications for human 
health. Using such spontaneous models, researchers have recently made significant discoveries on the interaction of papillomavirus with chemical carcinogens and immunosuppressants [4], on the role of BPV oncoproteins in cell transformation and immune evasion, on the infection of some unusual cell types and the possibility of haematogenous transplacental BPV transmission [5], as well as on new prophylactic and therapeutic vaccines [6-8].

In 2008, ten BPV types were recognized [9], but three new types have recently been proposed [10-12]. Bovine papillomavirus are grouped into three genera within the family Papillomaviridae: Deltapapillomavirus (BPV-1, BPV-2 and BPV-13), Xipapillomavirus (BPV-3, BPV-4, BPV-6, and BPV-9 to -12) and Epsilonpapillomavirus (BPV-5 and BPV-8), which show important inter-generic differences (e.g. Xipapillomavirus lack the E6 gene) [13] (Figure 1). The classification of BPV-7 remains to be determined [14] and there are at least sixteen other virus strains under study, detected in samples from Brazil, Japan and Sweden, which await classification, as recently reviewed by Zhu and co-workers [11]. In cattle, infection with several different BPV types is associated with the development of cutaneous, udder and teat papillomas and fibropapillomas [15-18] and cancer of various organ systems. In particular, BPV-4 causes upper digestive tract papillomas while BPV-2 and, less commonly, BPV-1 are associated with urinary bladder tumours [9]. Papillomavirus are strictly species-specific, with the remarkable exception of BPV-1 and, to a lesser extent, BPV-2. These two BPV types show a varied tropism for unusual cell types and even for species other than cattle, including equids such as horses, donkeys and zebras, where they cause fibroblast-derived cutaneous sarcoid tumours [19-20]. The following sections will deal with recent findings regarding BPV-induced tumours and the roles of BPV proteins, the interactions between BPV and chemical carcinogens, viral strategies to develop latency and enhance transmission, and new vaccination strategies. References to HPV and to other animal papillomavirus will be made when appropriate.

\section{BPV-induced cell transformation}

Tumours induced by BPV in cattle and horses provide a valuable opportunity for researchers to study the mechanisms through which viral oncoproteins - E5, E6 and E7 - transform infected cells. BPV E5 is a small, hydrophobic protein, mostly located in the in the Golgi apparatus and endoplasmic reticulum, and is considered a major transforming oncoprotein (reviewed by Venutti et al. [21]). Accordingly, its role in 
carcinogenesis has long been the focus of intense research. Many of HPV E5 functions have been first elucidated in BPV models, while some BPV E5 activities are not even shared by HPV E5, such as the activation of PDGFR $\beta$ and the tyrosine kinase c-src in bovine bladder carcinomas. Bovine papillomavirus type 2 and, less frequently, BPV-1 cause a non-productive infection of the urothelium in cattle, which has been associated with the development of urinary bladder tumours [22-23]. A similar pathology showing identical features was recently described in Turkish water buffaloes [24]. Several studies on bovine urothelial neoplasms reported the expression of cancer markers (reviewed by Gil da Costa et al. [4]) such as calpain-3, recently proposed to contribute to carcinogenesis by cleaving pRB [25]. Bovine urinary bladder tumours are often of mesenchimal or mixed mesenchimal/epithelial origin, often involving the endothelium (haemangiomas, haemagiosarcomas). Besides being present in the urothelium, BPV-2 DNA and oncoproteins (E5 and E7) have also been detected in neoplastic endothelial cells, where E5 was also shown to co-localize with PDGFR $\beta$ [26]. E5 is believed to be critical in driving cell transformation in vitro and in vivo, through several mechanisms, especially by activating the platelet-derived growth factor receptor $\beta$ (PDGFR $\beta$ ) [21]. As recently described, the binding of BPV-1 E5 to PDGFR $\beta$ results in activation of the phophatidylinositol-3-kinase (PI3K)-AKT-cyclin D pathway, leading to cell-cycle deregulation [27]. In higher-grade urothelial carcinomas PDGFR $\beta$ recruits growth factor receptor-bound protein 2 (GRB2) and its constitutively associated protein Sos, leading to RAS activation, but not to downstream Erk or Mek phosphorylation [28]. Based on these findings, the PI3K-AKT-cyclin D rather than the RAS-Erk pathway seems to be instrumental in driving papillomavirus-induced urothelial carcinogenesis (Figure 2).

The roles of E6 and E7 oncoproteins have also been studied in BPV-induced tumours. Importantly, BPV-1 E6 (together with HPV-1 and -8 E6) has been recently reported to bind the mastermind-like protein 1 (MAML1), repressing the NOTCH pathway and consequent gene transcription [29]. Based on these results, the authors propose that delayed keratinocyte differentiation in papillomavirus-induced papillomas may be a consequence of impaired NOTCH signalling. Despite lacking the canonical pRBbinding motif, BPV E7 plays a significant role in bovine and equine tumours. Infection of the bovine upper gastrointestinal mucosa by BPV-4 leads to papillomatosis of variable extension and severity. Upon prolonged exposure to bracken (Pteridium spp., a carcinogenic fern) papillomas progress to squamous cell carcinomas, providing a useful 
example of viral and chemical co-carcinogenesis [30]. As recently reviewed by Gil da Costa et al., [4] malignant transformation seems to depend on the mutagenic and immunosuppressant properties of bracken toxins which, on the one hand, promote genomic instability, resistance to apoptosis and cell-cycle deregulation by inactivating $p 53$ and activating $r a s$ and, on the other hand, prevent the immune system from clearing the viral papillomas (Figure 3). The bracken flavonoid quercetin, abundant in fruits and vegetables, also activates transcription from the BPV-4 long control region (LCR), up-regulating E7 expression and contributing to cell transformation in vitro [13]. Bearing in mind that, in human populations, HPV infection has been demonstrated in a significant proportion of oesophageal cancer cases and that bracken exposure is epidemiologically associated with higher oesophageal cancer risk, the hypothesis has been advanced that a similar mechanism may underlie oesophageal carcinogenesis in humans [1]. As with gastrointestinal tumours, bracken toxins are thought to drive the malignant transformation of initial bladder viral lesions. However, the interaction between BPV and bracken is not as clear as in the gastrointestinal tract. In fact, ptaquiloside from bracken is capable of inducing pre-neoplastic and neoplastic bladder lesions in laboratory animals alone, without any viral involvement [4, 31]. Bovine bladder tumours have attracted great interest not only due to their implication for animal health and the dairy industry, but also because they constitute a valuable spontaneous model to study the role of viral oncoproteins in cell transformation.

BPV E7 is also thought to be important in preventing anoikis, possibly by binding to the retinoblastoma protein-associated factor p600 in vitro. Binding of p600 has now also been reported in naturally occurring equine sarcoids, bringing additional support to this hypothesis [32]. Sarcoids are locally aggressive tumoural lesions that develop in equids (horses, donkeys, zebras) from dermal fibroblasts that harbour a latent, most commonly non-productive BPV-1 or BPV-2 infection and maintain the BPV-1/2 genomes in an episomal form [20]. Much interest has been focused on the mechanisms through which BPV-1 and -2 transform equine fibroblasts, causing equine sarcoids. Interestingly, the number of viral DNA copies has been shown to be significantly higher in more aggressive sarcoids, compared with their slowgrowing counterparts [33]. Over the years, the expression of several cancer markers has been studied in equine sarcoids, such as the tumour suppressor gene fragile histidine triad (FHIT), which was found to be down-regulated [34] as in human cervical cancer [35] although, curiously, not in BPV-associated bovine urinary tumours [36]. Such differences make spontaneous, 
BPV-induced, animal cancer models particularly useful to study the influence of specific host's genes in the carcinogenic process. Over-expression of matrix metalloproteinases (MMP) is an important mechanism involved in tumourigenesis, and was recently studied in detail at the University of Glasgow. MMP-1, -2 and -9 are over-expressed in sarcoid fibroblasts and decreased expression of viral genes by E2 siRNA treatment effectively reduced MMP expression, demonstrating the link between viral infection and MMP expression [37]. In particular, MMP-1 overexpression was shown to be driven by E6 and E7 through the activator protein-1 (AP-1) transcription factor [38], and by E5 through an indirect mechanism. However, only E5 and E6 were found to confer invasiveness and enhanced proliferation to equine fibroblasts in vitro, while E7 was associated with anchorage-independent growth [39]. E5 and E6 induce the over-expression of p38 mitogen-activated protein kinase (MAPK) which in turn leads to phosphorylation of an AP-1 component (Fra-1), triggering MMP-1 expression. Both increased proliferation and invasiveness were shown to be mediated by MAPK [40]. The MAPK-AP-1-MMP-1 signalling cascade was thus shown to be involved in the invasiveness of equine sarcoid fibroblasts.

BPV-mediated immune evasion

Evading the host's innate and adaptive immune responses is essential for maintaining viral infection. BPV E5 helps blocking the interactions between infected cells and their surrounding environment, especially by irreversibly downregulating major histocompatibility complex class I (MHC I) expression. This is achieved in a variety of ways, including the disruption of protein assemblage and traffic at the Golgi apparatus, thus preventing MHC I from being displayed on the cell surface [21]. Impaired protein oligomerization in the Golgi apparatus was also the mechanism proposed to explain connexin 43 down-regulation and the loss of intercellular gap junctions in bovine bladder carcinomas (Figure 2) [41]. This loss of gap junctions is likely to contribute to make infected cells refractory to inhibitory signals from neighbouring cells.

BPV-1 was also the first papillomavirus found to down-regulate the expression of the Toll-like receptor 4 (TLR4) through its oncoproteins E2 and E7, using transfected equine fibroblasts cultures in vitro [42]. TLRs are involved in triggering the innate immune response and, accordingly, TLR4 down-regulation is expected to contribute to immune evasion and viral persistence. 
Recent findings show how ptaquiloside, the main bracken carcinogen, helps inhibiting the innate immune response. Ptaquiloside was shown to enhance the expression of metallothienin-1 and -2 in natural killer (NK) cells, resulting in lower intracellular zinc concentrations and in NK function down-regulation [43-44] (Figure 3). One of the functions of NK cells is to destroy virus-infected cells and certain tumour cells. In the context of papillomavirus infection, this immunosuppressive effect is likely to promote viral persistence and tumour development. The same group demonstrated that NK cell function can be restored upon selenium administration [45]. These findings highlight the importance of environmental immunosuppressants, such as bracken, in the pathogenesis of papillomavirus-induced tumours.

\section{BPV persistence, latency and transmission}

The E2 protein associates with the host's chromatin and with the viral genome during mitosis, allowing viral maintenance in dividing cells. The actual mechanisms through which the E2 protein tethers papillomaviral episomes to host chromosomes are the subject of intense debate [46]. Using synchronised live cells, Feeney et al. [47] have shown that the DNA helicase ChlR1 is required for the association of BPV-1 E2 with chromosomes. In their recent paper, the authors proposed that ChIR1 is required for loading the E2-associated viral genomes onto chromosomes during the S-phase, but not for their retention during mitosis.

The transcriptional regulator functions of E2 proteins have also been studied using BPV. A recent study describes the different regulator activities of the full-length E2 protein (E2-TA) and of its two truncated isoforms, E2-TR and E8E2 [48], both of which have been described as transcriptional repressors. This study shows that, while E8E2 indeed acts as a transcription repressor, E2-TR acted as a transcriptional activator of E2dependent promoters. However, in contrast to E2-TA, E2-TR activated transcription from a promoter-proximal position and partially inhibited P89, while E8E2 led to complete repression.

Latent BPV-2 infection of peripheral blood lymphocytes resulting in chromosomal aberrations has long been known to occur in cattle [49-50], However, investigations on viral activity and the expression of viral proteins in peripheral blood mononuclear cells (PBMCs) have only started. Hartl et al. [51] reported that foals challenged intradermally with BPV-1 develop viraemia during the early stages of tumourigenesis. Viral DNA was detected in PMBCs before pseudo-sarcoids become palpable, at day 11-32 post- 
challenge, and the blood viral load correlates with tumour size. Moreover, E5 mRNA was demonstrated in PMBCs, indicating that viral oncogenes are expressed in these cells. Roperto et al. [52] also reported BPV-2 E5 expression in monocytes, Blymphocytes and, especially, in $\mathrm{CD}^{+}$and $\mathrm{CD} 8^{+} \mathrm{T}$-lymphocytes from cattle with urinary bladder tumours. These two cell populations were the only ones to also show expression of the L1 protein, associated with productive infections, and the authors accordingly concluded that they represent the most important BPV-2 blood reservoir.

In a recent study, viral DNA has also been found in the epidermis of horses with inflammatory skin conditions and in the epidermis overlying sarcoids, especially in early-stage, "occult", flat lesions [53]. Results suggest that a transient, productive infection may take place in the overlying epidermis during the early phases of sarcoid development [54-55]. These important findings raise the possibility of BPV transmission between equids, through direct contact or through fomites.

The presence and replication of BPV-2 in the bovine placenta has also been recently demonstrated [5]. Viral particles were demonstrated by electron microscopy in both endometrial and chorionic epithelial cells. Expression of E2 and E1 was confirmed, together with that of E5, which co-localized with PDGFR. $\beta$ Although there was already some data to support placental infection by HPV [56-57], these important findings confirm that papillomavirus are capable of establishing a productive infection in placental cells in vivo, and bring additional support to the hypothesis that children can be infected by HPV in utero.

Prophylactic and therapeutic vaccines

Innovative prophylactic and therapeutic vaccination strategies for equine sarcoids and bovine urinary bladder tumours have recently been advanced, mostly based on the use of virus-like particles (VLPs). In 1996, Kirnbauer et al. [58] first reported in a seminal study, that VLPs comprising the BPV-4 L1 and L2 or only the L1 protein triggered a strong humoural response in calves. This response led to immunity on a subsequent challenge with BPV-4, but did not induce papiloma regression in previously infected animals [52].

Later on, chimeric VLPs comprising BPV-1 L1 and E7 proteins were tested on sarcoidbearing donkeys [59] and horses [60] for therapeutic purposes. Despite a robust humoural response against L1 [60] and a tendency for tumour regression in some animals, results were not conclusive. However, very recently, BPV-1 L1 VLPs were 
tested in a dose-escalation trial for the vaccination of healthy horses and were shown to induce a long-lasting humoural response, which the authors deemed appropriate for protection [8].

A binary ethylenimine-inactivated and saponized BPV-2 vaccine was also tested against BPV-associated bovine urinary bladder tumours, but no regression was obtained [7]. All these results agree in showing that current BPV vaccination strategies may be more useful for prophylactic rather than therapeutic purposes.

\section{Conclusions}

Research on BPV continues to be of use for elucidating the mechanisms underlying virally-induced cell transformation. Indeed, in order to study important topics such as the synergy between papillomavirus and bracken toxins, BPV-based models can hardly be put aside. Research on BPV is also expected to continue providing useful leads and clues concerning the functions of viral proteins, especially E2 and E5. Studies on the mechanisms of viral persistence and transmission through blood lymphocytes and the placenta are of particular interest, due to their implications for public health. Important information is also being gained regarding distressing animal pathologies with high economical impact, such as bovine oro-oesophageal and urinary bladder cancer or equine sarcoids. At the same time, the ever-growing number of known BPV types holds the promise of new and unexpected possibilities for studying the molecular biology of papillomavirus.

\section{References}

[1]- Campo MS (2002) Animal models of papillomavirus pathogenesis. Virus Res 89:249-261.

[2]- Wang H-K, Duffy AA, Broker TR, Chow LT (2009) Robust production and passaging of infectious HPV in squamous epithelium of primary human keratinocytes. Genes \& Dev 23:181-94.

[3]- Pinto CA (2010) Hematúria enzoótica bovina: contribuição para o seu estudo etiopatogénico. PhD Disseration, Faculty of Veterinary Medicine, Technical University of Lisbon. 
[4]- Gil da Costa RM, Bastos MMSM, Oliveira PA, Lopes C (2012) Bracken-associated human and animal health hazards: chemical, biological and pathological evidence. $\mathbf{J}$ Hazard Mater 203-204:1-12.

[5]- Roperto S, Borzacchiello G, Esposito I, Riccardi M, Urraro C, Lucà R, Corteggio A, Taté R, Cermola M, Paciello O, Roperto F (2012) Productive infection of bovine papillomavirus type 2 in the placenta of pregnant cows affected with urinary bladder tumors. PloS One 7:e33569.

[6]- Pejawar-Gaddy S, Rajawat Y, Hilioti Z, Xue J, Gaddy DF, Finn OJ, Viscidi RP, Bossis I (2010) Generation of a tumor vaccine candidate based on conjugation of a MUC1 peptide to polyonicpapillomavirus virus-like particles (VLPs). Cancer Immunol Immunother 59:1685-1696.

[7]- Pathania S, Kumar P, Devi LG, Kumar D, Dhama K, Somvanshi R (2011) Preliminary assessment of binary ethylenimine inactivated and saponized cutaneous warts (BPV-2) therapeutic vaccine for enzootic bovine haematuria in hill cows. Vaccine 29:7296-7302.

[8]- Hainisch EK, Brandt S, Shafty-Keramat S, van den Hoven R, Kirnbauer R (2012) Safety and immunogenicity of BPV-1 L1 virus-like particles in a dose-escalation vaccination trial in horses. Equine Vet $\mathrm{J}$ 44:107-111.

[9]- Borzacchiello G, Roperto F (2008) Bovine papillomaviruses, papillomas and cancer in cattle. Vet Res 39:45.

[10]- Hatama S, Ishihara R, Ueda Y, Kanno T, Uchida I (2011) Detection of a novel papillomavirus type 11 (BPV-11) using xipapillomavirus consensus polymerase chain reaction primers. Arch Virol 156:1281-1285.

[11]- Zhu W, Dong J, Shimizu E, Hatama S, Kadota K, Goto Y, Haga T (2012) Characterization of novel bovine papillomavirus type 12 (BPV-12) causing epithelial papilloma. Arch Virol 157:85-91. 
[12]- Lunardi M, Alfieri AA, Otonel RA, de Alcântara BK, Rodrigues WB, de Miranda AB, Alfieri AF (2013) Genetic characterization of a novel bovine papillomavirus member of the Deltapapillomavirus genus. Vet Microbiol 162:207-13.

[13]- Campo MS (2006) Bovine papillomavirus: old system, new lessons? In: Campo MS (ed) Papillomavirus Research: from natural history to vaccines and beyond. Caister Academic Press, Wymondham, pp 373-87.

[14]- Ogawa T, Tomita Y, Okada M, Shirasawa H (2007) Complete genome and phylogenetic position of bovine papillomavirus type 7. J Gen Virol 88:1934-1938.

[15]- Rai GK, Saxena M, Singh V, Somvanshi R, Sharma B (2011) Identification of bovine papillomavirus 10 in teat warts of cattle by DNase-SISPA. Vet Microbiol 147:416-419.

[16]- Sá e Silva M, Weiss M, Brum MCS, dos Anjos BL, Torres FD, Weiblen R, Flores EF (2010) Molecular identification of bovine papillomaviruses associated with cutaneous warts in southern Brazil. J Vet Diagn Invest 22:603-606.

[17]- Hatama S, Nishida T, Kadota K, Uchida I, Kanno T (2009) Bovine papillomavirus type 9 induces epithelial papillomas on the teat skin of heifers. Vet Microbiol 136:347351.

[18]- Maeda Y, Shibahara T, Wada Y, Kadota K, Kanno T, Uchida I, Hatama S (2007)An outbreak of teat papillomatosis in cattle caused by bovine papilloma virus (BPV) type 6 and unclassified BPVs. Vet Microbiol 121:242-248.

[19]- Potocki L, Lewinska A, Klukowska-Rötzler J, Bugno-Poniewierska M, Koch C, Mählmann K, Janda J, Wnuk M (2012) DNA hypomethylation and oxidative stressmediated increase in genomic instability in equine sarcoid-derived fibroblasts. Biochimie 94:2013-2024. 
[20]- Nasir L, Reid SWJ (2006) Bovine papillomavirus and equine sarcoids. In: Campo MS (ed) Papillomavirus Research: from natural history to vaccines and beyond, Caister Academic Press, Wymondham, pp 389-398.

[21]- Venutti A, Paolini F, Nasir L, Corteggio A, Roperto S, Campo MS, Borzacchiello G (2011) Papillomavirus E5: the smallest oncoprotein with many functions. Mol Cancer 10:140.

[22]- Roperto S, Borzacchiello G, Brun R, Leonardi L, Maiolino P, Martano M, Paciello O, Papparella S, Restucci B, Russo V, Salvatore G, Urraro C, Roperto F (2010a) A review of bovine urothelial tumours and tumour-like lesions of the urinary bladder. $\mathbf{J}$ Comp Pathol 142:95-108.

[23]- Resendes AR, Roperto S, Trapani F, Urraro C, Rodrigues A, Roperto F, Borzacchiello G (2011) Association of bovine papillomavirus type 2 (BPV-2) and urinary bladder tumours in cattle from the Azores archipelago. Res Vet Sci 90:526-529.

[24]- Roperto S, Russo V, Ozkul A, Sepici-Dincel A, Maiolino P, Borzacchiello G, Marcus I, Esposito I, Riccardi MG, Roperto F (2013) Bovine papillomavirus type 2 infects the urinary bladder of water buffalo (Bubalus bubalis) and plays a crucial role in bubaline urothelial carcinogenesis. J Gen Virol 94:403-408.

[25- Roperto S, de Tullio R, Raso C, Stifanese R, Russo V, Gaspari M, Borzacchiello G, Averna M, Paciello O, Cuda G, Roperto F (2010b) Calpain3 is expressed in a proteolitically active form in papillomavirus-associated urothelial tumors of the urinary bladder in cattle. PLoS One 22:e10299.

[26]- Borzacchiello G, Russo V, Spoleto C, Roperto S, Balcos L, Rizzo C, Venutti A, Roperto F (2007) Bovine papillomavirus type-2 DNA and expression of E5 and E7 oncoproteins in vascular tumours of the urinary bladder in cattle. Cancer Lett 250:8291.

[27]- Corteggio A, Urraro C, Roperto S, Roperto F, Borzacchiello G (2010) Phosphatidylinositol-3-kinase-AKT pathway, phospho-JUN and phospho-JNK 
expression in spontaneously arising bovine urinary bladder tumours. J Comp Pathol 143:173-178.

[28]- Corteggio A, di Geronimo O, Roperto S, Roperto F, Borzacchiello G (2012) Activated platelet-derived growth factor $\beta$ receptor and Ras-mitogen-activated protein kinase pathway in natural bovine urinary bladder carcinomas. Vet J 191:393-395.

[29]- Brimer N, Lyons C, Wallberg AE, Vande Pol SB (2012) Cutaneous papillomavirus E6 oncoproteins associate with MAML1 to repress transactivation and NOTCH signalling. Oncogene 31:4639-4646.

[30]- Lucena RB, Rissi DR, Kommers GD, Pierezan F, Oliveira-Filho JC, Macedo JT, Flores MM, Barros CS (2011) A retrospective study of 586 tumours in brazilian cattle. J Comp Pathol 145:20-24.

[31]- Gil da Costa RM, Oliveira PA, Vilanova M, Bastos MMSM, Lopes CC, Lopes C (2011) Ptaquiloside-induced B-cell lymphoproliferative and early-stage urothelial lesions in mice. Toxicon 58:543-549.

[32]- Corteggio A, di Geronimo O, Roperto S, Roperto F, Borzacchiello G (2011b) Bovine papillomavirus E7 oncoprotein binds to p600 in naturally occurring equine sarcoids. J Gen Virol 92:378-382.

[33]- Haralambus R, Burgstaller J, Klukowska-Rötzler J, Steinborn R, Buchinger S, Gerber V, Brandt S (2010) Intralesional bovine papillomavirus DNA loads reflect severity of equine sarcoid disease. Equine Vet J 42:327-331.

[34]- Strazzullo M, Corteggio A, Altamura G, Francioso R, Roperto F, D’Esposito M, Borzacchiello G (2012) Molecular and epigenetic analysis of the fragile histidine triad in equine sarcoids. BMC Vet Res 8:30.

[35]- Garnieri E, Zanesi N, Bottoni A, Alderidio M, Lukic A, Vecchione A, Ziparo V, Croce CM, Mancini R (2010) Oncosuppresor proteins of fragile sites are reduced in cervical cancer. Cancer Lett 89:40-45. 
[36]- Guidi E, Uboldi C, Ferretti L (2008) Molecular analysis of the fragile histidine triad (FHIT) tumour suppressor gene in vesical tumors of cattle with chronic enzootic hematuria (CEH). Cytogenet Genome Res 120:173-177.

[37]- Yuan Z, Gobeil PAM, Campo MS, Nasir L (2010b) Equine sarcoid fibroblasts over-express matrix metalloproteinases and are invasive. Virology 396:143-151.

[38]- Yuan Z, Gault EA, Campo MS, Nasir L (2011b) Upregulation of equine matrix metalloproteinase 1 by bovine papillomavirus type 1 is through the transcription factor activator protein-1. J Gen Virol 92:2608-2619.

[39]- Yuan Z, Gault EA, Campo MS, Nasir L (2011a) Different contribution of bovine papillomavirus type 1 oncoproteins to the transformation of equine fibroblasts. $\mathrm{J}$ Gen Virol 92:773-783.

[40]- Yuan Z, Gault EA, Campo MS, Nasir L (2011c) p38 mitogen-activated protein kinase is crucial for bovine papillomavirus type- 1 transformation of equine fibroblasts. $\mathrm{J}$ Gen Virol 92:1778-1786.

[41]- Corteggio A, Florio J, Roperto F, Borzacchiello G (2011a) Expression of gap junction protein connexin 43 in bovine urinary bladder tumours. J Comp Pathol 144:8690.

[42]- Yuan Z, Bennet L, Campo MS, Nasir L (2010a) Bovine papillomavirus type 1 E2 and E7 proteins down-regulate Toll like receptor 4 (TLR4) expression in equine fibroblasts. Virus Res 149:124-127.

[43]- Latorre AO Furlan MS, Sakai M, Fukumasu H, Hueza IM, Haraguchi M, Górniak SL (2009) Immunomodulatory effects of Pteridium aquilinum on natural killer cell activity and on select aspects of the cellular immune response of mice. J immunotoxicol 6:104-114. 
[44]- Latorre AO, Caniceiro BD, Fukumasu H, Gardner DR, Lopes FM, Wysochi HL Jr, da Silva TC, Haraguchi M, Bressan FF, Górniak SL (2013) Ptaquiloside reduces NK cell activities by enhancing metallothionein expression, which is prevented by selenium. Toxicology 304:100-8.

[45]- Latorre AO, Carniceiro BD, Wysoki Jr. HL, Haraguchi M, Gardner DR, Górniak SL (2011) Selenium reverses Pteridium aquilinum-induced immunotoxic effects. Food Chem Toxicol 49:464-470.

[46]- You J, Croyle JL, Nishimura A, Ozato K, Howley PM (2004). Interaction of the bovine papillomavirus E2 protein with Brd4 tethers the viral DNA to host mitotic chromosomes. Cell 117:349-360.

[47]- Feeney KM, Saade A, Okrasa K, Parish JL (2011) In vivo analysis of the cell cycle dependent association of the bovine papillomavirus E2 protein and ChIR1. Virology 414:1-9.

[48]- Lace MJ, Ushikai M, Yamakawa Y, Anson JR, Ishiji T, Turek LP, Haugen TH (2012) The truncated C-terminal E2 (E2-TR) protein of bovine papillomavirus (BPV) type- 1 is a transactivator that modulates transcription in vivo and in vitro in a manner distinct from the E2-TA and E8E2 gene products. Virology 429:99-111.

[49]- Stocco dos Santos RC, Lindsey CJ, Ferraz OP, Pinto JR, Mirandola RS, Benesi FJ, Birgel EH, Pereira CA, Beçak W (1998) Bovine papillomavirus transmission and chromosomal aberrations: an experimental model. J Gen Virol 79:2127-2131.

[50]- Melo TC, Diniz N, Campos SR, Ferraz OP, Lindsey CJ, Rieger TT, Beçak W, Stocco RC (2011) Cytogenetic studies in peripheral blood of bovines afflicted by papillomatosis. Vet Comp Oncol 9:269-274.

[51]- Hartl B, Hainisch EK, Shafti-Keramat S, Kirnbauer R, Corteggio A, Borzacchiello G, Tober R, Kainzbauer C, Pratscher B, Brandt S (2011) Inoculation of young horses 
with bovine papillomavirus type 1 virions leads to early infection of PBMCs prior to pseudo-sarcoid formation. J Gen Virol 92:2437-2445.

[52]- Roperto S, Comazzi S, Ciusani E, Paolini F, Borzacchiello G, Esposito I, Lucà R, Russo V, Urraro C, Venuti A, Roperto F (2011) PBMCs are additional sites of productive infection of bovine papillomavirus type 2. J Gen Virol 92:1787-1794.

[53]- Wobeser BK, Hill JE, Jackson ML, Kidney BA, Mayer MN, Townsend HGG, Allen AL (2012) Localization of bovine papillomavirus in equine sarcoids and inflammatory skin conditions of horses using laser microdissection and two forms of DNA amplification. J Vet Diagn Invest 24:32-41.

[54]- Bogaert L, Martens A, Kast WM, van Marck E, de Cock H (2010) Bovine papillomavirus DNA can be detected in keratinocytes of equine sarcoid tumors. Vet Microbiol 146:269-275.

[55]- Brandt S, Tober R, Corteggio A, Burger S, Sabitzer S, Walter I, Kainzbauer C, Steinborn R, Nasir L, Borzachiello G (2011) BPV-1 infection is not confined to the dermis but also involves the dermis of equine sarcoids. Vet Microbiol 150:35-40.

[56]- Sarcola ME, Grénman SE, Rintala MA, Syrjänen KJ, Syrjänen SM (2008) Human papillomavirus in the placenta and umbilical cord blood. Acta Obstet Gynecol Scand 87:1181-1188.

[57]- Weyn C, Thomas D, Jani J, Guizani M, Donner C, van Rysselberge M, Hans C, Bossens M, Englert Y, Fontaine V (2010) Evidence of human papillomavirus in the placenta. J Infect Dis 203:341-343.

[58]- Kirnbauer R, Chandrachud LM, O’Neill BW, Wagner ER, Grindlay GJ, Armstrong A, McGarvie GM, Schiller JT, Lowy DR, Campo MS (1996) Virus-like particles of bovine papilomavírus type 4 in prophylactic and therapeutic immunization. Virology 219:37-44. 
[59]- Ashrafi GH, Piuko K, Burden F, Yuan Z, Gault EA, Müller M, Trawford A, Reid SWJ, Nasir L, Campo MS (2008) Vaccination of sarcoid-bearing donkeys with chimeric virus-like particles of bovine papillomavirus type-1. J Gen Virol 89:148-157.

[60]- Mattil-Fritz S, Scharner D, Piuko K, Thönes N, Gissmann L, Müller H, Müller M (2008) Immunotherapy of equine sarcoid: dose-escalation trial for the use of chimeric papillomavirus-like particles. J Gen Virol 89:138-147. 


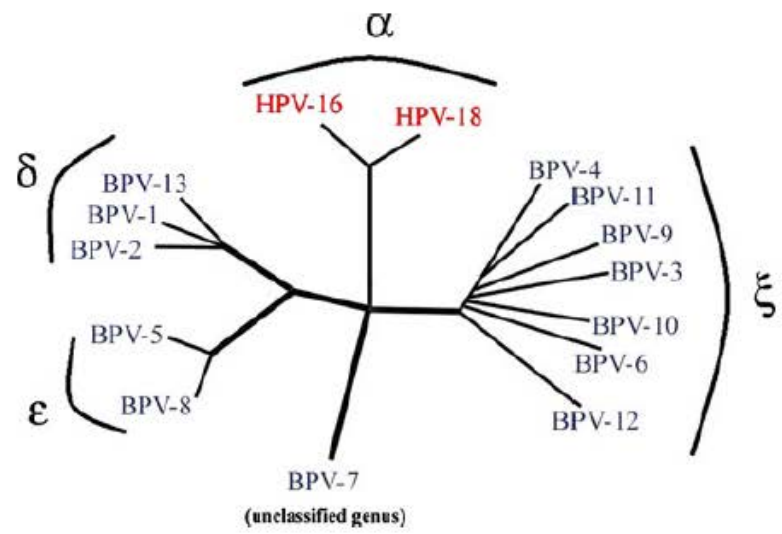

Figure 1- Neighbour-joining phylogenetic tree of bovine papillomaviruses (BPV, blue) and some high risk human papillomaviruses (HPV, red), based on the L1 nucleotide sequence. Greek letters indicate the genus.

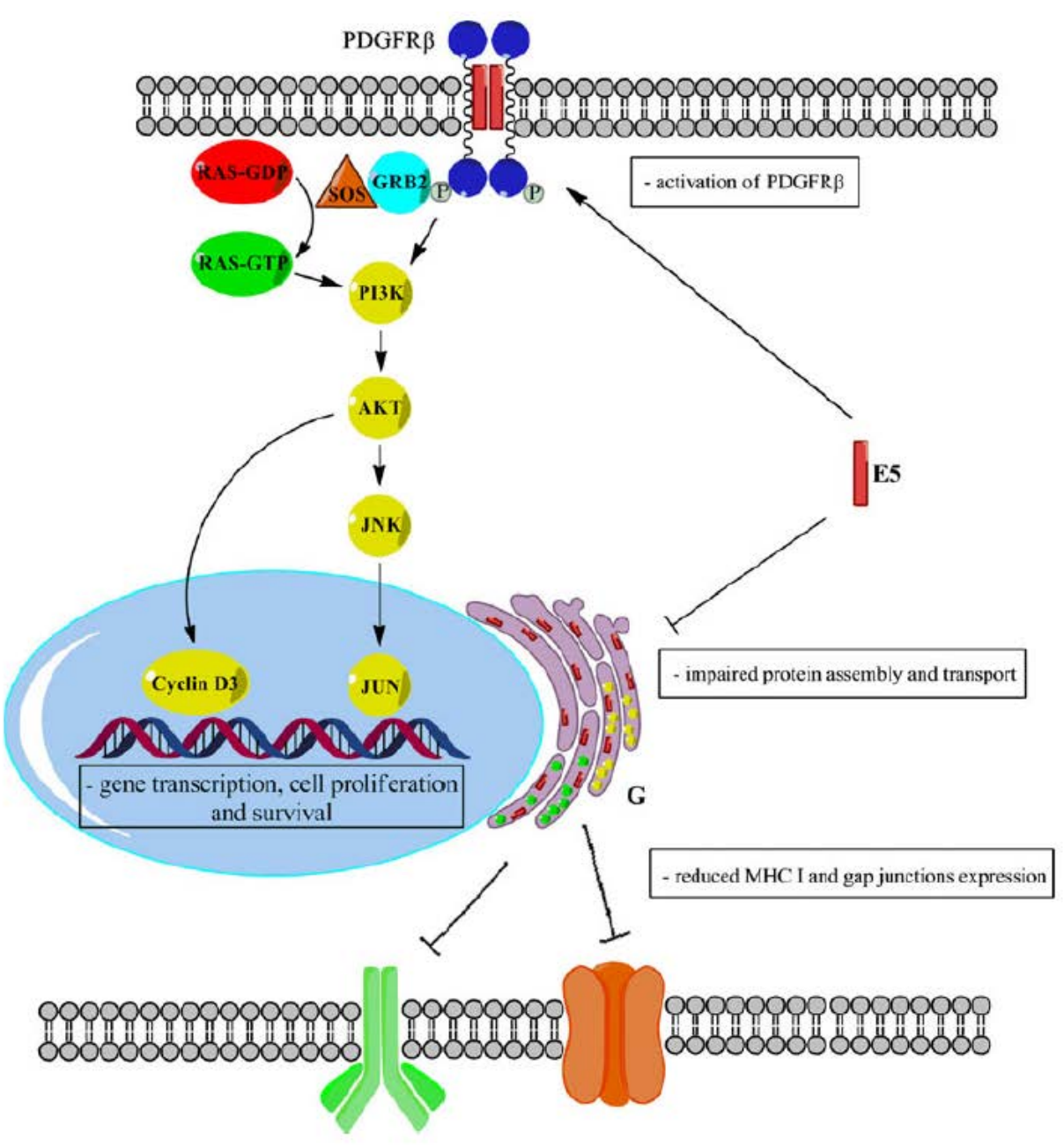

Figure 2- Some of the BPV-1 E5 oncogenic actions on PDGFR and the Golgi apparatus (G), in bovine urothelial carcinomas. E5 activates PDGFR , which triggers the PI3K-AKT pathway and recruits the GRB2-SOS, activating RAS. AKT up-regulates cyclin D and is proposed to activate JNK, leading to altered gene expression. In the Golgi apparatus, E5 blocks MHC I (green) and connexin 43 (yellow) assembly and traffic, down-regulating surface MHC I and gap junctions. 


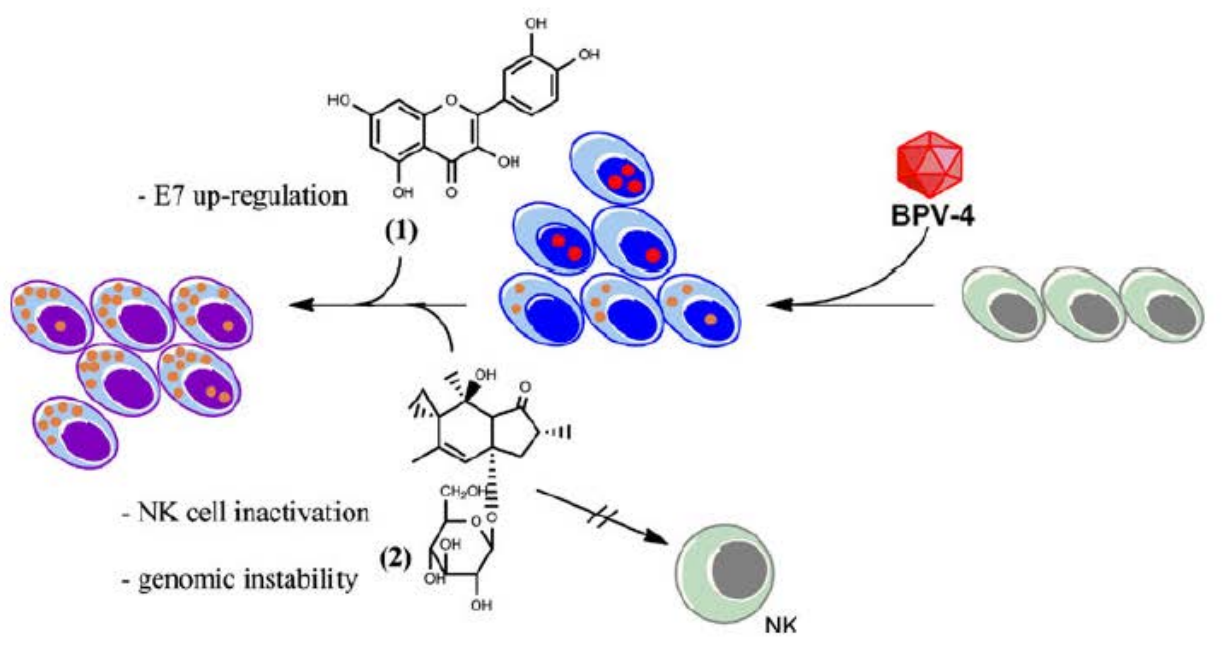

Figure 3- Interaction between BPV-4 and some bracken toxins in upper digestive carcinogenesis. BPV-4 infects normal epithelial cells (grey), initiating exophytic proliferations (papillomas, in blue), which show virion production (red) and E7 oncoprotein expression (yellow). In a second phase, quercetin (1) is proposed to up-regulate E7 expression while ptaquiloside (2) blocks natural killer (NK) cell function, reducing immunosurveillance, and contributes to genomic instability, promoting carcinogenesis and leading to oro-oesophageal carcinomas (violet). 